Historia y comunicación social

ISSN: 1137-0734

\title{
Gobernanza del sistema mediático cubano. Un estudio del marco regulatorio sobre comunicación desde 1959 hasta 2018
}

\author{
Aimiris Sosa Valcárcel ${ }^{1}$; Rina M. Aguilera Hintelholher²; Andrea Castro-Martínez
}

Recibido el: 9 de agosto de 2019 / Aceptado: 15 de julio de 2020

Resumen. Esta investigación caracteriza el sistema mediático cubano, a partir del estudio del marco regulatorio instaurado en el país durante cerca de 60 años. Se utiliza un diseño metodológico mixto de dos etapas para realizar un análisis de la normativa, a través del software cualitativo Atlas.ti. Los resultados indican que la regulación mediática es desarrollada principalmente por los poderes ejecutivo y político, y que la garantía de los derechos fundamentales relacionados con los medios de comunicación -la libertad de expresión, la autonomía editorial, el derecho a la información, o la independencia del sistema regulador- se encuentra sujeta a las condiciones que establece el sistema único de propiedad estatal y al control que sobre los medios ejerce el partido comunista.

Palabras clave: sistema mediático; regulación; poder; Estado; Cuba.

[en] Governance of the Cuban media system. A study of the regulatory framework on communication from 1959 to 2018

\begin{abstract}
This research characterizes the Cuban media system, based on the study of the regulatory framework established in the country for nearly 60 years. A mixed methodological design of two stages is used to perform an analysis of the regulations through Atlas.ti qualitative software. The results indicate that the media regulation is mainly developed by the executive and political powers, and that the guarantee of fundamental rights related to the media -freedom of expression, editorial autonomy, the right to information, or the independence of the regulatory system-is subject to the conditions established by the single system of state ownership and to the control over the media exercised by the Communist Party.
\end{abstract}

Keywords: media system; regulation; power; State; Cuba.

Sumario: 1. Introducción. 1.1. Papel del estado en la regulación de los medios. 1.2. El caso Cuba. 2. Metodología. 3. Resultados. 3.1. Normativa cubana sobre los medios de comunicación. 3.2. Elementos de la comunicación que aparecen regulados. 3.3. Principios reguladores del servicio público y participación de las audiencias. 4. Discusión y conclusiones. 5. Bibliografía.

Cómo citar: Sosa Valcárcel, A.; Aguilera Hintelholher, R. M.; Castro-Martínez, A. (2021) Gobernanza del sistema mediático cubano. Un estudio del marco regulatorio sobre comunicación desde 1959 hasta 2018, Historia y comunicación social 26(1), 95-105.

\section{Introducción}

Desde los inicios de la sociedad moderna los sistemas mediáticos aparecen estrechamente vinculados al poder político (Reig y Labio, 2017; Domínguez, 2018). Las características y potencialidades de los medios masivos permiten la construcción y socialización de símbolos funcionales de dominación y cambio social (Murdock, 2017; Bezerra, 2018), que la clase política necesita para el establecimiento y la reproducción del poder (Castells, 2008; Mico y Carbonell, 2017).

\footnotetext{
Universidad de Málaga

E-mail: aimirissosa@uma.es

ORCID: https://orcid.org/0000-0001-8480-8063

2 Universidad Nacional Autónoma de México

E-mail: rinaaguilera@politicas.unam.mx

Universidad de Málaga

E-mail: andreacastro@uma.es

ORCID: https://orcid.org/0000-0002-2775-625X
}

Hist. comun. soc. 26(1) 2021: 95-105 
El recurso a los medios de comunicación se erige como condición sine qua non para la formación de la opinión pública y la toma de decisiones políticas (De Aguilera y Casero, 2018), sobre todo en el escenario actual, donde las Tecnologías de la Información y la Comunicación (TIC) condicionan los procesos de producción, distribución y consumo de productos comunicativos a escala global (Gershon, 2016).

Esta investigación analiza el marco normativo que ha regulado el sistema de medios de comunicación en Cuba, durante cerca de 60 años. Se pretende con ello determinar cómo la práctica regulatoria cubana ha definido "las condiciones en que las diferentes fuerzas establecen relación en cuanto a la comunicación, así como, en caso de conflicto, las instancias y condiciones del arbitraje que debe ejercer el Estado" (Fox, 1975: 94). Y es que, como asegura Pérez (2014: 52), Cuba carece de "una estructura jurídica que apoye una Política Nacional que contribuya al derecho a la información y todo lo que se deriva de su reconocimiento legal". De ahí la pertinencia del estudio que aquí se realiza.

La investigación sobre sistemas mediáticos y sus vínculos con la política ha sido desarrollada por diferentes paradigmas de la ciencia: desde las Four Theories of the Press, en las que Siebert, Peterson y Schramm (1956: 1) aseguran que "la prensa toma la forma y la coloración de las estructuras sociales y políticas en las que opera"; pasando por la propuesta de Martín-Serrano (1986) que explica la interdependencia entre medios y política, a partir de la mediación que ejercen en dichos sistemas la infraestructura tecnológica de la sociedad, sus estructuras de relaciones sociales y la supraestructura ideológica.

Resultan relevantes además las aportaciones de Luhmann (1998) que en su teoría de sistemas coloca a la comunicación como el elemento constitutivo de la sociedad. El autor considera al sistema mediático como un subsistema que proporciona estabilidad al sistema social, en tanto produce y reproduce objetos que surgen de la comunicación. Alude también a la capacidad de los medios para agendar los temas relevantes que conciernen al poder político y reconocerlos como públicos (Luhmann, 2000).

Entre los autores paradigmáticos de la teoría social contemporánea se encuentra Habermas (1987), quien destaca el rol que el sistema mediático desempeña en las sociedades democráticas, en tanto evita "la captura o el monopolio político, social o económico de los foros de la comunicación pública" (Habermas, 2009: 39).

Thompson (2002), por otra parte, ofrece una perspectiva fundamental para entender el papel político de la comunicación de masas, cuando establece el principio del pluralismo regulado en la organización y operación de las instituciones de los medios. Para la fundamentación de este principio el autor hace un recorrido por dos teorías precedentes -la doctrina liberal tradicional de la prensa libre (Mill, 1970; Saavedra, 1987; Milton, 2009), y el principio de la radiodifusión de servicio público (Reith, 1924) - a las cuales reconoce sus limitaciones: de un lado, la concentración comercial y, de otro, la interferencia estatal en el desarrollo de los sistemas mediáticos.

Propone entonces "situar las instituciones de los medios en el espacio existente entre el mercado y el Estado" (Thompson, 2002: 380-381), lo cual exige una desconcentración de recursos en las industrias de los medios, a través de una legislación que limite las actividades de los conglomerados de la comunicación; y una separación clara de las instituciones de los medios del ejercicio del poder estatal.

Se trata, en suma, de la formulación de políticas que generen una democratización real de la comunicación (Mcbride, 1980; Díaz, 1981; Beltrán, 2006; Mattelart, 2007; Bustamante, 2014; Sierra, Barragán y Moreno, 2018; Gumucio, 2019), y que favorezcan la confluencia de medios en un espacio público plural "cuyos protagonistas en el Estado y en la esfera de la ciudadanía, movilicen los instrumentos de la democracia en contra de las apropiaciones simbólicas mediante los valores mercantiles" (De Moraes, 2011: 133).

Ello conlleva a concebir la información y la comunicación como derecho ciudadano que corresponde al Estado garantizar (De Frutos, 2014), en tanto organización encargada de crear las condiciones para impulsar las actividades productivas, así como asegurar el orden y la seguridad en cada uno de los puntos del territorio que le corresponde salvaguardar

\subsection{Papel del estado en la regulación de los medios}

En un sentido de acción inteligente, el Estado no constituye un problema para la sociedad, sino condición necesaria para su desarrollo. Sin desconocer la importancia del mercado y el Estado para la vida de la sociedad, no debe plantearse su relación en la lógica fallida que confronta al Estado con el mercado, sino vincularlos con otras capacidades productivas de la sociedad, en una visión de cooperación y reconocimiento de cada una de las entidades mencionadas (Kliksberg, 2001: 24).

El Estado tiene a su cargo capitalizar las aportaciones que desde la vida privada, la sociedad y la vida pública pueden y deben hacer los actores que dan cuenta de la pluralidad y diversidad que hay en la lógica del desarrollo eficaz (Gutiérrez, Restrepo y Zapata, 2017). Los fines colectivos del Estado se cumplen de manera práctica e interrelacionada, lo cual implica "que las políticas públicas son parte de las políticas gubernamentales porque representan decisiones en donde participa el gobierno. Las políticas públicas son políticas gubernamentales con vasta intervención social" (Arias y Herrera, 2012: 46).

La misión del Estado, en consecuencia, es de amplia regulación (Majone y la Spina, 1993). Constituye un sistema de interacciones que tiene como objetivo producir e intercambiar recursos, información, bienes 
y servicios para garantizar el desarrollo de la vida en sociedad, a partir de instrumentos como las políticas gubernamentales (Amantino y Dias, 2017). Este activo escaso es de vital importancia para que la regulación, entendida como la vigencia de reglas institucionales, tenga como referente anticipado y previsible las normas que se deben cumplir, así como los derechos que se tienen para hacer efectiva las libertades democráticas (Mathieu, Verhoest y Matthys, 2017).

En el escenario actual de convergencia tecnológica, el acceso y la participación de los ciudadanos en la gestión de los asuntos públicos constituyen un requisito fundamental de las sociedades democráticas (Ramanzini y de Souza, 2016; Clinger, 2017). Las audiencias exigen hoy no sólo una mayor interacción con los medios, sino un rol mucho más activo en el sistema de control de la comunicación pública (Azurmendi, Llorens, López y Bas, 2015; Muñiz, Téllez y Saldierna, 2017)

En consecuencia, las políticas de gobierno que se aplican a los sistemas mediáticos se centran en la regulación de aspectos como estructura, infraestructura y tecnología, distribución, acceso, conducta y contenido (Mastrini, 2014), los cuales hacen referencia, entre otras, a cuestiones de: propiedad, finanzas, forma y organización en general, concentración, monopolio, condiciones de concesión de licencias y el control público o privado de los medios de comunicación; desarrollo, funcionamiento de las redes básicas de transmisión y asignación de frecuencias; servicios que se ofrecen y obligaciones impuestas a los proveedores; comportamiento de las organizaciones de los medios de comunicación, ética, derechos de autor, derecho a la información, seguridad del Estado y control de los contenidos (Hernández, 2015).

Los productos de la regulación para entenderla como política de gobierno a nivel nacional se relacionan con leyes, decretos, códigos, estatutos, reglamentos y acciones que se emprenden para que sean efectivas en la vida social y política, y que "pueden provenir de un consenso, cuando se trata de un Estado democrático, o bien ser formuladas de manera unilateral por gobernantes autocráticos" (Toussaint, 2011: 92-93).

Esta condición obliga a retomar el debate sobre cuestiones como pluralismo, diversidad, libertad de expresión, derecho a la comunicación (UNESCO, 2008; Girard, 2015), que corresponden al Estado preservar en beneficio del interés general (Santana, 2015; Pardo, 2016; Uvalle, 2018) y de la prestación de los servicios públicos (Bonet, Caamaño, Gimeno, Quintero y Sala, 2017).

Para Mastrini (2011) los principios organizadores de los servicios públicos son igualdad/universalidad, continuidad y cambio; y significan que la política pública debe garantizar a la ciudadanía en condiciones de igualdad la disponibilidad de los servicios, su prestación de forma constante, así como su necesaria actualización.

Sin desconocer que los sistemas mediáticos presentan características diferentes en función de los países donde operan, existe consenso, desde la teoría, de la necesidad de contar con marcos regulatorios que garanticen el desarrollo de procesos de comunicación pública transparentes, participativos y democráticos, en los que intervengan todos los actores sociales (Salazar, 2019). La práctica, en cambio, demuestra que en el contexto actual de predominio de medios comerciales y concentración de la propiedad, la preservación de estas garantías a nivel global continúa siendo una asignatura pendiente.

\subsection{El caso Cuba}

El sistema mediático cubano, objeto de estudio de esta investigación, se encuentra compuesto por más de 600 medios oficiales entre los que se incluyen periódicos, emisoras de radio, canales de televisión, revistas impresas, agencias de información y sitios nativos digitales. Todos son de propiedad estatal y surgieron a partir del 1 de enero de 1959 (gráfico 1).

Gráfico 1. Tipología de medios de comunicación. Cuba, 2018

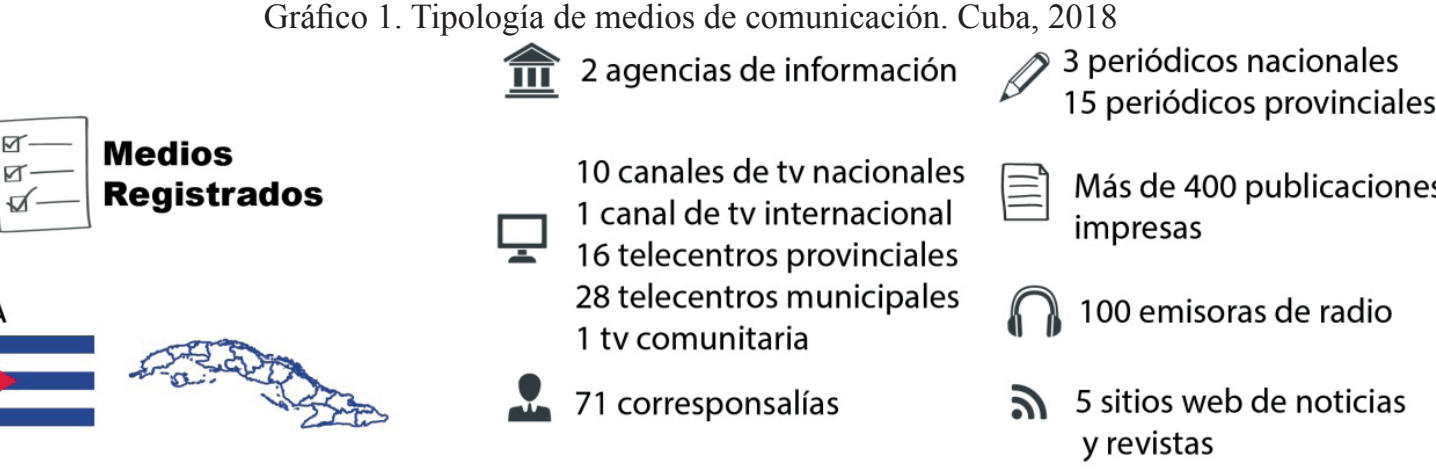

Fuente: Elaboración propia. 
Desde entonces la regulación del sector ha estado vinculada a las condiciones históricas en las que se han producido "los principales cambios institucionales, de relaciones políticas, normativos, ideológico-culturales y comunicativos del país" (Duharte, 2005: 5).

Entre las características fundamentales del sistema de medios Somohano (2013: 83) reconoce "la condición social de la propiedad y el sustento en el principio de que la información no debe someterse a intereses privados, ni a procesos de comercialización". Estas premisas si bien han demostrado la capacidad del Estado para salvaguardar el interés público, justifican al mismo tiempo la permanencia de "un modelo comunicativo que antepone el sentido de compromiso político por sobre el concepto de libertad de expresión, a la hora de establecer las directrices de obligado cumplimiento para la producción y el consumo de los contenidos" (Autor, 2019: 149).

Para García (2013) este es uno de los principales obstáculos del sistema de medios de comunicación en Cuba, en el que se aprecian "las fuerzas de resistencia interna, tanto por la inercia de la costumbre, como por posiciones basadas en convicciones sinceras, por dogmatismos o por la sublimación de intereses de poder" (García, 2013: 174).

Con la llegada de Internet el debate sobre la regulación de los medios adquiere una complejidad mayor, a partir de la emergencia de publicaciones que funcionan fuera del sistema oficial de comunicación controlado por La Habana y, por tanto, al margen del ordenamiento jurídico nacional (gráfico 2). Elizalde (2013: 156) advierte sobre la necesidad de evitar que:

la denominada "revolución digital" termine ausente de las políticas públicas y autorregulada por el mercado, como proyecto ideológico por el cual el Estado se hace cargo del pasado mientras deja el futuro de la comunicación y la cultura en manos del mercado.

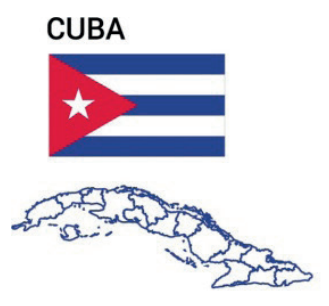

Gráfico 2. Medios no registrados. Cuba, 2018

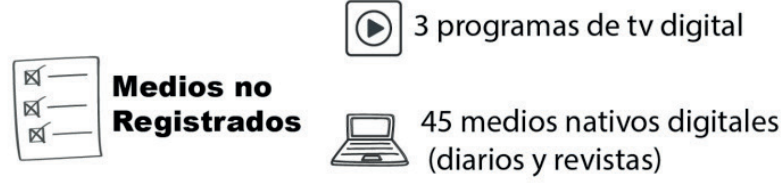

Fuente: Cubaposible. Elaboración propia.

En este escenario el presente estudio se plantea como objetivos:

1. Identificar las disposiciones legales que en torno a los medios de comunicación han estado vigentes en Cuba entre 1959 y 2018.

2. Analizar los elementos de la comunicación que aparecen regulados en la normativa cubana.

3. Describir la forma en que se expresan los principios reguladores del servicio público.

4. Determinar el rol que el marco regulatorio cubano confiere a las audiencias en el sistema de rendición de cuentas de los medios.

\section{Metodología}

Esta investigación descriptiva se desarrolla a partir de un diseño metodológico mixto de dos etapas en el que se aplica, en primer lugar, un enfoque cualitativo para explorar y analizar el contenido de las disposiciones legales que en torno al sistema mediático cubano han estado vigentes entre 1959 y 2018. En un segundo momento se cuantificó los datos cualitativos obtenidos y se les asignó códigos a las categorías. El número de veces que cada código aparece fue registrado como dato numérico (Hernández, Fernández y Baptista, 2010).

Teniendo en cuenta el amplio marco temporal que abarca el estudio, no interesa en este caso realizar un relato cronológico de la normativa, pues algunas disposiciones extienden su período de vigencia por décadas. Se trata de exponer los principales ejemplos en torno a la regulación de los medios, desde la perspectiva de los elementos de la comunicación que son planificados y regulados, y de los principios definitorios del servicio público que aparecen en la legislación.

En el período que abarca la investigación se encontraron 92 disposiciones legales sobre los medios de comunicación. Este universo de normas fue estudiado mediante el software de análisis cualitativo Atlas.ti, que permite crear unidades hermenéuticas donde se recogen, organizan y codifican los documentos asociados a un objeto de estudio (Gallardo, 2014). 
Para el análisis se utilizó una codificación deductiva, a partir de la creación de una lista de 30 códigos agrupados en seis categorías: tipo de norma, ente regulador, elementos de la comunicación regulados, principios organizadores del servicio público, participación de las audiencias y sanciones (gráfico 3).

Gráfico 3. Categorías y códigos de análisis

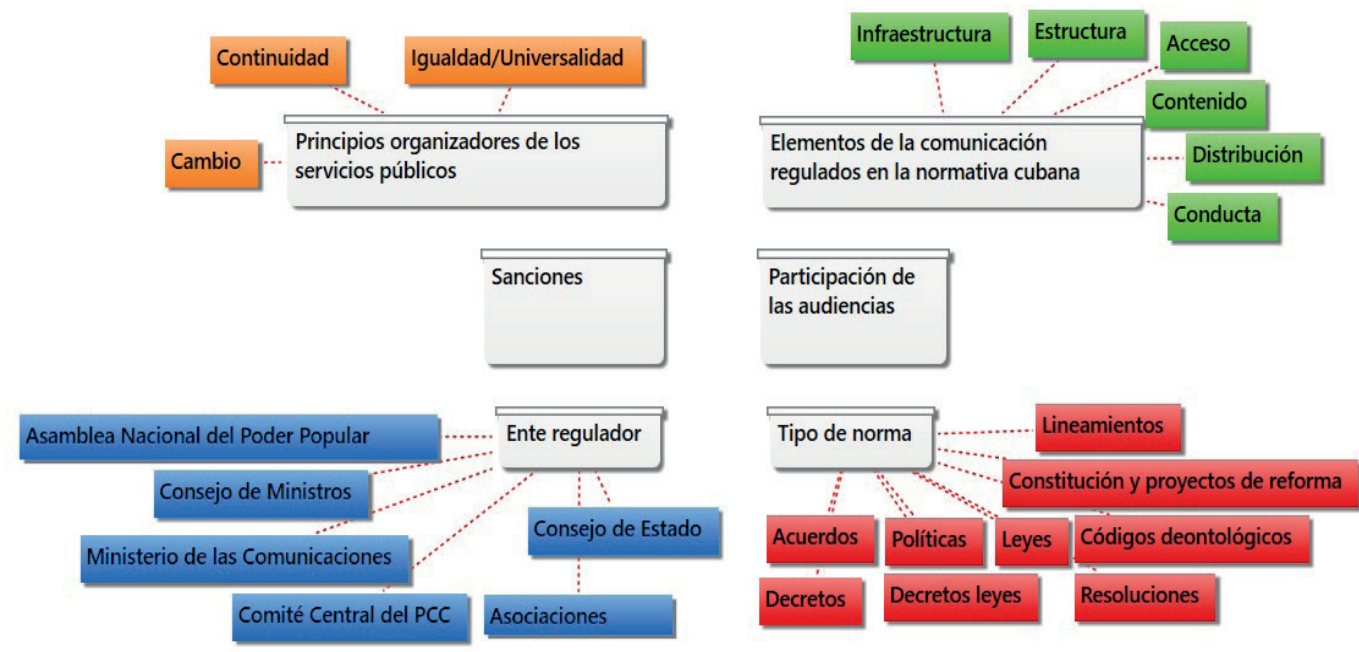

Fuente: Elaboración propia.

\section{Resultados}

\subsection{Normativa cubana sobre los medios de comunicación}

El marco regulatorio de los medios de comunicación en Cuba está compuesto por una serie de normas expresadas en: la Constitución de la República y sus proyectos de reforma, leyes, decretos-leyes, decretos, acuerdos, resoluciones, códigos deontológicos, políticas organizacionales y los lineamientos que rigen la política económica y social del país.

La aprobación de dichas normas ha estado a cargo de organismos reguladores como la Asamblea Nacional del Poder Popular (Legislativo), los Consejos de Estado y de Ministros y el ministerio con competencias en comunicación (Ejecutivo), el Comité Central del Partido Comunista de Cuba (Político), así como la Unión de Periodistas de Cuba y la Asociación de Comunicadores Sociales de Cuba (Gremial) (gráfico 4).

Gráfico 4. Relación entre normativa y ente regulador de la comunicación. Cuba (1959-2018)

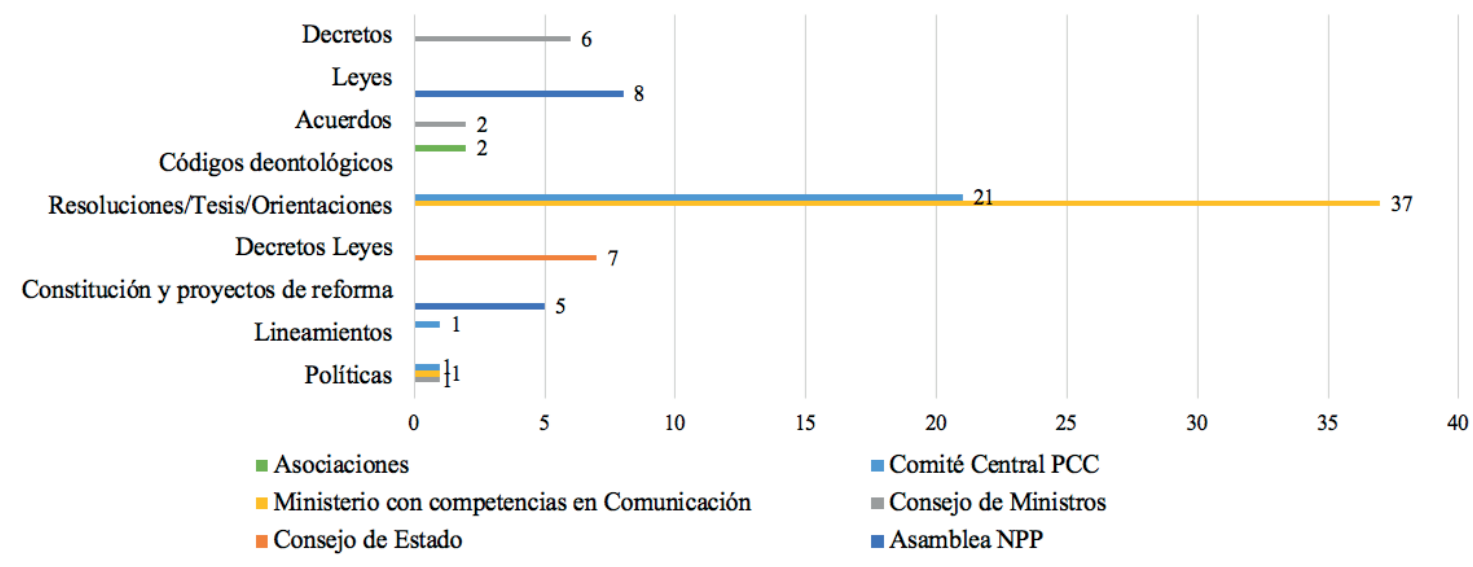

Fuente: Elaboración propia.

El análisis refleja que el $76 \%$ de la normativa ha sido regulada por los poderes ejecutivo y político, y sólo un $24 \%$ corresponde al poder legislativo y a las asociaciones que representan los intereses del gremio. Esta centralidad en la regulación de los medios evidencia que no todos los actores sociales han tenido las mismas 
oportunidades para participar en el debate público, lo cual asegura un entorno mediático parcializado, poco favorable para el ejercicio eficaz de la transparencia y la participación ciudadana en la rendición de cuentas.

Del total de regulaciones estudiadas, el $24,4 \%$ dispone sanciones ante el incumplimiento total o parcial de los términos y condiciones requeridas. Aunque la mayoría de estas sanciones se establecen a nivel administrativo y comprenden, entre otras, multas por uso inadecuado del espectro radioeléctrico (Decreto n. 171, 1992), inhabilitación en el ejercicio de la radioafición (Decreto n. 188, 1994), separación o expulsión del gremio periodístico (UPEC, 2017); el código penal también reconoce determinados supuestos constituyentes de delito, relacionados con los medios de comunicación. El artículo 103.1 establece que:

Incurre en sanción de privación de libertad de diez a quince años el que utilice los medios de difusión masiva para incitar contra el orden social, la solidaridad internacional o el Estado socialista, mediante la propaganda oral o escrita o en cualquier otra forma; o para difundir noticias falsas o predicciones maliciosas tendentes a causar alarma, descontento en la población, o desorden público (Ley n. 62, 1987: 37).

\subsection{Elementos de la comunicación que aparecen regulados}

En los documentos estudiados destaca la estructura como el elemento de la comunicación al que se alude con mayor frecuencia. La normativa reconoce de forma explícita que los medios de comunicación son propiedad del Estado, así como "el servicio de radiodifusión, sonora y de televisión es estatal y no comercializado, esencial para llevar la información, la educación y el entretenimiento a toda la sociedad" (Resolución n. 175, 2013: 819).

Sujeto a este principio de propiedad estatal aparece regulado el derecho ciudadano a la libertad de palabra y prensa:

Las condiciones materiales para su ejercicio están dadas por el hecho de que la prensa, la radio, la televisión, el cine y otros medios de difusión masiva son de propiedad estatal o social y no pueden ser objeto, en ningún caso, de propiedad privada, lo que asegura su uso al servicio exclusivo del pueblo trabajador y del interés de la sociedad (Constitución del República de Cuba, 1976: 14).

La carta magna aprobada en Cuba en 2019 añade que el Estado "establece los principios de organización y funcionamiento para todos los medios de comunicación social" (Constitución del República de Cuba, 2019: 81), y que ejerce soberanía y jurisdicción sobre el espectro radioeléctrico.

De manera general la normativa cubana que regula la estructura dispone las condiciones de uso del espectro radioeléctrico "por los organismos de la economía nacional y los encargados de la defensa y seguridad del país", asumiendo que se trata de un "recurso natural limitado" necesario para "el desarrollo y la construcción del socialismo" (Decreto n. 135, 1986: 1).

En los textos analizados también se explicitan indicaciones sobre infraestructura, que si bien se refieren, por ejemplo, a la solicitud de "licencias y autorizaciones para la instalación y operación de las estaciones de radiocomunicaciones requeridas para la prestación de los servicios concesionados" (Decreto n. 321, 2013: 89), o a la prohibición de fabricación e importación de "equipos transmisores y transceptores por las personas naturales y jurídicas" sin previa autorización de los organismos competentes (Decreto n. 135, 1986: 5); se centran además en hacer balance de las infraestructuras existentes y las que se planifican de cara al futuro, formuladas en su mayoría como deseos o pretensiones.

Es el caso de la Política de Comunicación Social del Estado y el Gobierno cubanos (2018: 18) que incluye en sus acciones "priorizar la infraestructura, conectividad y acceso a las TIC e impulsar la convergencia digital en los medios de comunicación masiva".

La conducta es otro elemento de la comunicación que se aborda en la normativa estudiada. Se encuentran recomendaciones e instrucciones sobre el comportamiento que deben seguir los medios de comunicación en cuestiones como la ética, los derechos ciudadanos respecto de los medios, o las conductas que se esperan de los comunicadores. Un ejemplo de ello es el código de ética de la Unión de Periodistas de Cuba (UPEC), donde se recoge que "el periodista contribuye con su trabajo a promover los mejores valores nacionales, el cabal conocimiento de las leyes y el perfeccionamiento constante de nuestra sociedad socialista" (2014:2).

La UPEC es una organización gremial con estatutos y régimen sancionador propios que recoge normas de conducta periodística. Algunas de ellas son: no hacer publicidad con forma de información periodística, no publicar declaraciones o datos de fuentes con la advertencia explícita de no ser publicadas, o respetar el secreto estatal vigente en Cuba.

El contenido es uno de los elementos que recaba mayor número de referencias en la regulación cubana. Gran parte de ellas resultan recomendaciones realizadas por el Comité Central del Partido Comunista de Cuba (CCPCC), sobre las líneas a seguir para favorecer el sistema político instaurado y para promover la conciencia socialista, dada la importancia de los medios de difusión masiva en la construcción del ideario político. Así lo reconocen en el primer congreso de la organización: 
El contenido de la radio, la televisión, la prensa escrita y el cine responde a su esencia clasista, cuyo carácter está determinado por el régimen de propiedad sobre estos medios, que, en ningún caso, pueden actuar al margen o por encima de las clases, sino que constituyen instrumentos de la lucha ideológica y política (CCPCC, 1975: 1).

En el caso de la distribución, el marco regulatorio apunta que los servicios de los medios de comunicación deben hacerse extensivos a todo el pueblo, e indica la gama que se ofrecen y la forma en que se distribuyen a lo largo del territorio nacional.

Para la prensa impresa establece, por ejemplo, que Correo es la empresa estatal encargada de la "recepción, distribución, transporte y entrega de los envíos de correspondencia y bultos postales en general, impuestos dentro del territorio nacional y dirigido a localidades del mismo territorio" (Decreto n. 3.508, 1965: 5).

El servicio de distribución del sistema de radiofrecuencias corresponde a la Empresa de Radiocomunicación y Difusión de Cuba (RADIOCUBA), único proveedor en el país que garantiza las transmisiones de las señales de radio y televisión, con la salida al aire de las emisoras y canales de alcance internacional, nacional y local. Mientras, la Empresa de Telecomunicaciones de Cuba S.A. (ETECSA) se encarga, entre otras cuestiones, de la distribución de las redes públicas de telecomunicaciones y su uso "para transportar señales o dar servicios a terceros" (Decreto n. 321, 2013: 1).

Sobre la cuestión de acceso a los medios, la normativa regula los derechos de propiedad y operación, claramente definidos en el artículo 55 de la Constitución (2019), y explicitados en los casos concretos de acceso a los servicios de radioaficionados (Decreto n. 188, 1994), a la información oficial clasificada (DecretoLey n. 199, 1999) y "al empleo de las nuevas Tecnologías de la Informática" (Política integral para el perfeccionamiento de la informatización de la sociedad en Cuba, 2017: 3).

Este elemento de la comunicación aparece también condicionado al criterio del Consejo de Defensa Nacional, que en situaciones excepcionales como el estado de guerra, la guerra, la movilización general o el estado de emergencia "podrá regular de manera diferente el ejercicio de derechos fundamentales", entre los que se incluye la libertad de palabra y prensa (Ley n. 75, 1994: 2).

\subsection{Principios reguladores del servicio público y participación de las audiencias}

En la normativa cubana los principios de igualdad/universalidad, continuidad y cambio se sustentan en el sistema de propiedad estatal establecido sobre los medios de comunicación y en la directiva que sobre ellos ejerce el partido comunista, en términos de garantizar el desarrollo, la revisión y la actualización de los servicios que los medios ofrecen.

Sobre estas bases se encuentran disposiciones en las que se expresa el principio de igualdad/universalidad, cuando se refieren a garantizar a los usuarios los servicios de telecomunicaciones (Decreto n. 321, 2013), o a reconocer el derecho de las personas a participar en la vida cultural de la nación (Constitución de la República de Cuba, 2019).

Por otra parte, los principios de continuidad y cambio aparecen estrechamente relacionados, en tanto la actualización -estructural, tecnológica, de contenidos- de los servicios que ofrecen los medios, garantiza la estabilidad y permanencia del sistema político.

Es el caso de la remodelación técnica de la prensa, del sistema nacional de radiodifusión y televisión y de "todas las publicaciones escritas del Partido, la Unión de Jóvenes Comunistas y las organizaciones de masas" (CCPCC, 1985: 54); el desarrollo del sector de la informatización como "arma para la defensa de la Revolución y la ciberseguridad frente a las amenazas, los riesgos y ataques de todo tipo" (Política integral para el perfeccionamiento de la informatización de la sociedad en Cuba, 2017: 3); o "la aprobación del estándar de televisión terrestre como parte de la ejecución de la política nacional orientada a fortalecer el papel de las telecomunicaciones en el desarrollo político, económico y social del país" (Acuerdo n. 7.455, 2013: 1).

La participación de las audiencias en la formulación y el control de las políticas públicas que rigen a los medios de comunicación resulta poco tratada en la normativa cubana, con un $4,4 \%$ de representación. De los elementos de la comunicación previamente analizados, la regulación sólo se refiere al contenido y a la estructura como objetos factibles en los que la sociedad civil puede participar.

Para ejercer la participación en los contenidos se establecen como vías la correspondencia, secciones especializadas, entrevistas, encuestas, encuentros de las audiencias con los representantes de los órganos de dirección de los medios; corresponsales voluntarios en los centros de trabajo, de estudios y en las organizaciones de masas; o mediante la realización de encuentros entre los trabajadores de los medios y obreros, campesinos, combatientes o estudiantes.

Es deber de la radio, la televisión, la prensa escrita y el cine en nuestra sociedad, contribuir a que las masas no sean sólo receptores pasivos de un mensaje sino que consideren a los distintos órganos como vehículos para expresar sus criterios, sus sugerencias y quejas (CCPCC, 1975: 12). 
En términos de estructura el Decreto n. 188 (1994) reconoce la posibilidad de que existan estaciones de radioaficionados previamente autorizadas, que usen la radiofrecuencia con carácter exclusivamente personal y sin fines de lucro.

En ningún caso se concretan los modos de participación del público en los mecanismos de decisión y control sobre los medios, ni se reconoce el derecho a crear canales alternativos descentralizados para el debate sobre la regulación del sistema mediático.

\section{Discusión y conclusiones}

La regulación es un tipo de política gubernamental que tiene por objeto definir las reglas del juego que se aplican en la sociedad sobre la base de incentivos y sanciones. Alude a formas de intervención que la autoridad constituida realiza para que los conflictos, los intereses y las soluciones de la vida colectiva se desarrollen en ámbitos de gobiernos organizados en diversos sistemas de jurisdicción, que establecen los modos y formas para que los cuerpos burocráticos y los mandos políticos actúen en razón de las normas escritas.

El Estado es la suma de poderes que se instituyen para generar las capacidades de gobierno que hacen posible esa regulación de la vida colectiva con fines de producción, reproducción y un mejor rendimiento, que conlleven a garantizar la formación de la riqueza material y su distribución con procesos y estrategias de gobierno. Su función es de relevancia medular en tanto organizador de las condiciones que posibilitan la concurrencia de la producción y los servicios públicos que se distribuyen entre los agentes productivos y los sectores de la vida social.

Tal y como establece la UNESCO (2008: 5), un componente indispensable de los servicios públicos que ofrecen los Estados es el desarrollo de entornos mediáticos en los cuales "puedan prosperar la libertad de expresión, independencia y pluralismo de los medios". Ello es posible mediante el establecimiento de un sistema regulador que garantice -constitucional y jurídicamente- el acceso y la participación de la ciudadanía en las políticas públicas de comunicación.

En el caso cubano, como en otros contextos que se han dado desde los inicios de la sociedad moderna (Reig y Labio, 2017; Domínguez, 2018), el sistema mediático aparece estrechamente vinculado a las élites de poder; con la peculiaridad de que en esta relación se otorga legalmente al poder político, ejercido por el partido comunista, una capacidad de dirección y control que en ocasiones se equipara al poder que representa la institución del Estado.

De esta forma se logra, coincidiendo con las aportaciones de Murdock (2017), construir símbolos funcionales de dominación a través de los medios de comunicación, los cuales legitiman el discurso del partido comunista, esto es, de la revolución, y se convierten en un recurso efectivo para la reproducción del poder (Castells, 2008; Mico y Carbonell, 2017).

En Cuba la regulación de los elementos de la comunicación gira en torno a la estructura de los medios, que se basa, coincidiendo con Somohano (2013), en la condición social de la propiedad y en la exclusión de intereses privados del ámbito de la información. En relación con ello aparecen explicitadas recomendaciones en cuanto a la conducta y los contenidos de los medios que, si bien suelen ser indicaciones en favor de la supraestructura ideológica, están formuladas de manera diversa. Estas indicaciones inciden en los valores del socialismo de modo que, tal y como indica Luhmann (2000), hacen públicos y difunden temas concernientes al poder político.

La normativa incluye también un repaso por las infraestructuras existentes, alabando los logros previos y efectuando previsiones de dotación que fortalezcan las condiciones de acceso. Así, en cuanto a la regulación de elementos de la comunicación, queda patente la carencia de una esfera pública plural (Habermas, 2009) propia de las sociedades democráticas. Además, pese a que se reconoce la participación de las audiencias, esto no se concreta de forma efectiva en ningún caso en cuanto a la decisión y control sobre los medios, ni existe en la legislación la posibilidad de crear canales alternativos para el debate sobre los asuntos públicos.

Los principios reguladores del servicio público se plantean de forma que la igualdad/universalidad aparecen enfocadas como parte del compromiso del sistema de propiedad estatal para con el pueblo cubano. Este mismo principio, vinculado a la legitimación y permanencia del sistema político, rige los aspectos de continuidad y cambio, destacando en la normativa la necesidad de una actualización para adaptarse, entre otras cuestiones, a las nuevas tecnologías.

En el análisis que esta investigación realiza se constata, como ya adelantaba Pérez (2014), que a pesar de la trayectoria de la práctica regulatoria cubana por cerca de seis décadas, Cuba aún carece de un marco jurídico que asegure a la comunicación como un derecho, con garantías explícitas para que "las organizaciones de la sociedad civil, las organizaciones mediáticas y el público en general puedan influir en las políticas públicas sobre los medios" (UNESCO, 2008: 11).

En la misma línea que indican investigaciones previas (Autor, 2019), queda patente que la libertad de expresión se supedita en Cuba al compromiso político, en lo que concierne a la creación y al consumo de contenidos, ya que la normativa contempla sanciones ante el uso de los medios de difusión masiva contra el 
orden social imperante, es decir, el Estado socialista. Esto se debe en parte a la fuerte presencia del CCPCC como ente regulador, a través de resoluciones, tesis, orientaciones, lineamientos y políticas.

Se concluye, por tanto, que en el caso cubano pese a que se cumplen los principios reguladores del servicio público, no se da la separación señalada por Thompson (2002) entre los medios y el ejercicio del poder estatal, ya que la regulación no permite el pluralismo en la organización del sistema mediático.

\section{Bibliografía}

Acuerdo n. 7.455, de 10 de septiembre de 2013. (2013). "Aprobar la adopción del estándar de trasmisión de televisión digital terrestre DTMB". La Habana, Cuba. Recuperado de https://www.gacetaoficial.gob.cu/codbuscadores.php

Amantino, J. y Dias, J. (2017). "Analysis of public action instrumentation from the perspective of the actor-network theory: social technology and rural education in Rondônia". Rev. Adm. Pública, 51(3), pp. 407-430.

Arias, D. y Herrera, H. (2012). Entre políticas gubernamentales y politicas públicas. Análisis del ciclo de las políticas de desarrollo del gobierno del Estado de Michoacán, México 2003-2010. Ciudad de México, México: Instituto Nacional de Administración Pública, A.C.

Azurmendi, A., Llorens, C., López, N. y Bas, J. (2015). "La participación del público como valor añadido de servicio público para la televisión de proximidad. Estudio de caso de La noche de, en ETB 2". Revista Latina de Comunicación Social, 70, pp. 490-518.

Beltrán, L. (2006). "La comunicación para el desarrollo en Latinoamérica: un recuento de medio siglo". Anagramas, 4(8), pp. 53-76.

Bezerra, B. (2018). Los dueños de las empresas de comunicación y la utilización politica de los medios en Brasil: el caso de los estados Bahía, Ceará y Río Grande del Norte (Tesis de Doctorado). Universidad Complutense de Madrid, Madrid, España.

Bonet, J., Caamaño, F., Gimeno, J., Quintero, G. y Sala, P. (2017). Servicios públicos e ideología: el interés general en juego. Barcelona, España: Profit Editorial.

Bustamante, E. (2014). "La democratización del sistema cultural y mediático español. Ante una situación de emergencia nacional”. En M. Chaparro (Ed.), Medios de proximidad: participación social y políticas públicas (pp. 21-34). Girona/ Málaga, España: Gorbs Edicions.

Castells, M. (2008). "Comunicación, poder y contrapoder en la sociedad red. Los medios y la política". Telos, 74, pp. $1-15$.

Clinger, J. (2017). "The kantian publicity principle and the transparency presumption in public affairs: when private citizens' participation becomes public knowledge". Public Integrity, 19(4), pp. 394-403.

Comité Central del Partido Comunista de Cuba. (1975). Tesis y resoluciones sobre los medios de difusión masiva. Recuperado de https://www.pcc.cu/es/i-congreso

Comité Central del Partido Comunista de Cuba. (1985). Informe central del III Congreso del Partido Comunista de Cuba. Recuperado de https://www.pcc.cu/es/iii-congreso

Constitución de la República de Cuba, de 24 de febrero de 1976. (1976). "Establece los fundamentos constitucionales del país en todos los ámbitos". La Habana, Cuba. Recuperado de https://www.gacetaoficial.gob.cu/codbuscadores.php

Constitución de la República de Cuba, de 10 de abril de 2019. (2019). "Establece los fundamentos constitucionales del país en todos los ámbitos". La Habana, Cuba. Recuperado de https://www.gacetaoficial.gob.cu/codbuscadores.php

De Aguilera, M. y Casero, A. (2018). “¿Tecnologías para la transformación? Los medios sociales ante el cambio político y social". Presentación. Icono 14, 16(1), pp.1-21.

Decreto n. 3.508, de 15 de septiembre de 1965. (1965). "Reglamento de los servicios de correos, giros postales y telégrafos". La Habana, Cuba. Recuperado de https:/www.mincom.gob.cu/es/documento-legal/decreto-3508-1965

Decreto n. 135, de 6 de mayo de 1986. (1986). "Sobre el uso de las frecuencias radioeléctricas". La Habana, Cuba. Recuperado de https://www.mincom.gob.cu/es/documento-legal/decreto-135-1986

Decreto n. 171, de 6 de mayo de 1992. (1992). "Establece las contravenciones personales de las regulaciones del uso del espectro radioeléctrico". La Habana, Cuba. Recuperado de https://www.mincom.gob.cu/es/documento-legal/ decreto-171-1992

Decreto n. 188, de 5 de agosto de 1994. (1994). "Sobre el servicio de radioaficionados". La Habana, Cuba. Recuperado de https://www.mincom.gob.cu/es/documento-legal/decreto-188-1994

Decreto n. 321, de 4 de diciembre de 2013. (2013). "Concesión Administrativa a ETECSA para la prestación de servicios públicos de telecomunicaciones". La Habana, Cuba. Recuperado de https://www.mincom.gob.cu/es/documento-legal/ decreto-321-2013

Decreto-Ley n. 199, de 2 de diciembre de 1999. (1999). "Sobre la seguridad y protección de la información oficial". La Habana, Cuba. Recuperado de https://www.gacetaoficial.gob.cu/codbuscadores.php

De Frutos, R. A. (2014). "Mediciones enredadas. La importancia de los indicadores mediático-culturales para el análisis de los sistemas mediáticos". Historia y Comunicación Social, 190, pp. 525-537.

De Moraes, D. (2011). La cruzada de los medios en América Latina. Gobiernos progresistas y políticas de comunicación. Buenos Aires, Argentina: Paidós. 
Díaz, J. (1981). "Democratización de la comunicación: teoría y práctica". Chasqui: Revista Latinoamericana de Comunicación, 1, pp. 13-21.

Domínguez, H. (2018). "Relaciones entre medios y política: de la función política a la mediatización del conflicto". Revista Internacional de Comunicación y Desarrollo, 8, pp. 107-118.

Duharte, E. (2005). Teoría y procesos políticos contemporáneos. La Habana, Cuba: Félix Varela.

Elizalde, R. (2013). El consenso de lo posible. Principios para una política de comunicación social cubana socialmente consistente y tecnológicamente sustentable en los escenarios perspectivos de regulaciones externas e internas (Tesis de Doctorado). Universidad de La Habana, La Habana, Cuba.

Fox, E. (1975). Políticas nacionales de comunicación en América Latina. San José de Costa Rica, Costa Rica: CIESPAL.

Gallardo, E. (2014). Cómo realizar análisis temático utilizando Atlas.ti. Recuperado de https://atlasti.com/2014/06/12/ como-realizar-analisis-tematico-utilizando-atlas-ti/

García, J. (2013). Revolución, socialismo, periodismo: La prensa y los periodistas cubanos ante el siglo XXI. La Habana, Cuba: Pablo de la Torriente.

Gershon, R. (2016). Digital media and innovation. Management and design. Strategies in communication. London, England: Sage.

Girard, C. (2015). "Making democratic contestation possible: public deliberation and mass media regulation". Policy Studies, 36(3), pp. 283-297.

Gumucio, A. (2019). "Información, comunicación y cambio de mentalidad: nuevas agendas para un nuevo desarrollo". Alcance: Revista Cubana de Información y Comunicación, 7(18), pp. 238-249.

Gutiérrez, J., Restrepo, R., y Zapata, J. (2017). "Formulación, implementación y evaluación de políticas públicas desde los enfoques, fines y funciones del estado". Revista CES Derecho, 8(2), pp. 333-351.

Habermas, J. (1987). Teoría de la acción comunicativa. Tomo II. Crítica de la razón funcionalista. Madrid, España: Taurus.

Habermas, J. (2009). ¡Ay, Europa! Madrid, España: Trotta.

Hernández, M. (2015). Políticas públicas de comunicación. Estudio comparativo de las legislaciones audiovisuales de Argentina y España (Tesis de Doctorado). Universidad de Salamanca, Salamanca, España.

Hernández, R., Fernández, C. y Baptista, P. (2010). Metodología de la investigación. Ciudad de México, México: McGraw Hill.

Kliksberg, B. (2001). El nuevo debate sobre el desarrollo y el rol del Estado. Mitos y realidades en la América Latina de hoy. Ciudad de México, México: Instituto Nacional de Administración Pública, A.C.

Ley n. 62, de 29 de diciembre de 1987. (1987). "Código penal". La Habana, Cuba. Recuperado de http://www. parlamentocubano.cu/?documento=codigo-penal-2

Ley n. 75, de 21 de diciembre de 1994. (1994). "De la defensa nacional”. La Habana, Cuba. Recuperado de https://www. gacetaoficial.gob.cu/codbuscadores.php

Luhmann, N. (1998). Sistemas sociales: lineamientos para una teoría general. Barcelona, España: Anthropos.

Luhmann, N. (2000). La realidad de los medios de masas. Ciudad de México, México: Anthropos y Universidad Iberoamericana.

Macbride, S. (1980). Un solo mundo, voces múltiples: comunicación e información en nuestro tiempo. París, Francia: FCE-UNESCO.

Majone, G. y la Spina, A. (1993). "El Estado regulador". Gestión y Política Pública, II(2), pp. 197-261.

Martín-Serrano, M. (1986). La producción social de comunicación. Madrid, España. Alianza Editorial.

Mastrini, G. (2011). "Medios públicos y derecho a la comunicación: una aproximación desde América Latina". Portal de la Comunicación InCom-UAB, pp. 1-7.

Mastrini, G. (2014). Las industrias culturales en Argentina (Tesis de Doctorado). Universidad Complutense de Madrid, Madrid, España.

Mathieu, E., Verhoest, K., y Matthys, J. (2017). "Measuring multi-level regulatory governance: Organizational proliferation, coordination, and concentration of influence". Regulation \& Governance, 11(3), pp. 252-268.

Mattelart, A. (2007). La mundialización de la comunicación. Barcelona, España: Paidós.

Mico, J. y Carbonell, J. (2017). "The catalan political process for independence: an example of the partisan media system". American Behavioral Scientist, 61(4), pp. 428-440.

Mill, J. (1970). Sobre la libertad. Madrid, España: Alianza Editorial.

Milton, J. (2009). Areopagítica. Ciudad de México, México: Universidad Nacional Autónoma de México.

Muñiz, C., Téllez, N. y Saldierna, A. (2017). "Political sophistication as a mediator in the relation between media consumption and citizen participation. Evidence from the O-S-R-O-R model”. Communication \& Society, 30(3), pp. $255-274$.

Murdock, G. (2017). "Encoding and decoding”. The International Encyclopedia of Media Effects, pp. 1-11.

Pardo, C. (2016). Una introducción a la administración pública. Ciudad de México, México: El Colegio de México.

Pérez, N. (2014). "El derecho a la información en Cuba: consideraciones éticas y jurídicas en un entorno cambiante". Bibliotecas. Anales de Investigación, 10, pp. 37-53. 
Política de Comunicación del Estado y el Gobierno cubanos, de enero de 2018 (2018). "Sintetiza el conjunto de objetivos, principios, normas y aspiraciones en el campo de la comunicación social en Cuba”. La Habana, Cuba. Recuperado de https://www.periodismodebarrio.org/2018/12/que-dice-la-nueva-politica-de-comunicacion-cubana/

Política integral para el perfeccionamiento de la informatización de la sociedad en Cuba, de julio de 2017. "Documento rector para el perfeccionamiento de la informatización de la sociedad en Cuba de manera segura y sostenible". La Habana, Cuba. Recuperado de https://www.mincom.gob.cu/es/node/2469

Ramanzini, H. y de Souza, R. (2016). "Participation and influence: Democratization and the shaping of a public policy in Brazil”. Latin American Policy, 7(1), pp. 106-125.

Reig, R. y Labio, A. (2017). El laberinto mundial de la información. Barcelona, España: Anthropos.

Reith, J. (1924). Broadcast over Britain. London, England: Hodder \& Stoughton.

Resolución n. 175, de 14 de mayo de 2013. (2013). "Establece los indicadores de calidad del servicio de radiodifusión analógica en onda media, frecuencia modulada y televisión". La Habana, Cuba. Recuperado de https://www. gacetaoficial.gob.cu/codbuscadores.php

Saavedra, M. (1987). La libertad de expresión en el Estado de Derecho. Entre la utopía y la realidad. Barcelona, España: Ariel.

Salazar, G. (2019). "Aliados estratégicos y los límites de la censura: el poder de las leyes para silenciar a la prensa". Revista mexicana de ciencias politicas y sociales, 64(235), pp. 495-522.

Santana, L. (2015). A reformar la administración pública: de la burocracia a la gobernanza. San Juan, Puerto Rico: Escuela Graduada de Administración Pública, Universidad de Puerto Rico.

Siebert, F., Peterson, T. y Schramm, W. (1956). Four theories of the press: the authoritarian, libertarian, social responsability, and soviet communist concepts of what the press should be and do. Urbana, IL: University of Illinois.

Sierra, F., Barragán, V. y Moreno, J. (2018). Derechos humanos, comunicación y luchas por la dignidad. Quito, Ecuador: Ediciones CIESPAL.

Somohano, A. (2013). Regulación comunicativa y aproximación mediática a actores politicos: notas para un debate desde el contexto cubano. Régimen de regulación comunicativa sobre mecanismos de aproximación de Granma y Juventud Rebelde a la UJC (Tesis de Maestría). Universidad de La Habana, La Habana, Cuba.

Thompson, J. (2002). Ideología y cultura moderna. Teoría crítica social en la era de la comunicación de masas. Ciudad de México, México: Universidad Autónoma Metropolitana.

Toussaint, F. (2011). "La política cultural y las televisoras públicas en México, Venezuela y Brasil”. Estudios sobre las Culturas Contemporáneas, XVII (33), pp. 91-111.

Unión de Periodistas de Cuba. (2014). Código de ética del periodista. Recuperado de http://www.cubaperiodistas.cu/ index.php/codigo-de-etica-del-periodista/

UNESCO. (2008). Indicadores de desarrollo mediático: marco para evaluar el desarrollo de los medios de comunicación social. París, Francia: UNESCO.

Unión de Periodistas de Cuba. (2017). Reglamento del código de ética. Recuperado de http://www.cubaperiodistast.co.cu/ index.php/codigo-de-etica-del-periodista/reglamento-del-codigo-de-etica-capitulo-i/

Uvalle, R. (2018). "El nexo entre políticas públicas y organizaciones complejas”. En A. De la Rosa y J. Contreras (Eds.), Organizaciones y políticas públicas: un campo en construcción. Reflexiones teóricas y evidencias empíricas (pp. $25-$ 54). Ciudad de México, México: Fontamara. 